\title{
Comparison of montage with conventional stereoscopic seven-field photographs for assessment of ETDRS diabetic retinopathy severity
}

\author{
Nam V. Nguyen 1,2,4, Erin M. Vigil1,2,3, Muhammad Hassan', Muhammad S. Halim', Sean C. Baluyot²,
} Hugo A. Guzman², Rubbia Afridi ${ }^{1}$, Diana V. Do ${ }^{1}$ and Yasir J. Sepah ${ }^{1 *}$ (D)

\begin{abstract}
Background: The ETDRS stereoscopic seven-field (7F) has been a standard imaging and grading protocol for assessment of diabetic retinopathy (DR) severity score in many clinical trials. To the best of our knowledge, the comparison between montage and stereoscopic 7F has not been reported in the literature. Therefore, the main purpose of this study is to compare agreement between montage and stereoscopic seven-field (7F) photographs in the assessment of DR severity.

Methods: Stereoscopic 7F photographs were captured from subjects with DR. Montages of monoscopic $7 F$ images were created using Adobe Photoshop CS6 Extended@. The best quality image of each stereo pair was selected and placed on a $150 \times 125$-inch canvas field according to the standard location from field 1 to 7 . All the fields were aligned following the vessels and overlaid using the built-in blending tool. The resulting montage was utilized for grading and compared with grading on stereoscopic 7F photographs. Three independent graders were asked to assess DR severity on stereoscopic 7F photographs and montage. Severity level agreement between stereo $7 \mathrm{~F}$ and montage was crosstabulated and the agreement of DR severity levels between stereoscopic 7-field images and montage was analyzed using $\mathrm{k}$ intergrader agreement; statistical significance was set at $\mathrm{p}<0.05$.

Results: A total of 50 eyes were included in the study. There was a substantial agreement between stereoscopic $7 \mathrm{~F}$ and montage $\left(k=0.745, k_{\text {weighted }}=0.867\right)$ in assessment of DR severity. Of 50 eyes, $80 \%$ of the cases showed complete agreement, and $100 \%$ of the cases had agreement within one-step. There was a moderate agreement among graders, and $\mathrm{k}$-value ranged from 0.4705 to 0.5803 .
\end{abstract}

Conclusion: In this study, we found a substantial agreement in assessing DR severity score employing non-stereoscopic montage and stereoscopic $7 \mathrm{~F}$ photographs.

Keywords: Diabetic retinopathy severity score, Stereoscopic seven-field, Montage

\section{Background}

Diabetic retinopathy (DR), an ocular complication of diabetes, is the leading cause of irreversible blindness among Americans from age 20 to 74 years and accounts

\footnotetext{
*Correspondence: yjs@stanford.edu

${ }^{1}$ Byers Eye Institute, Stanford University, 2370 Watson Court, Suite 200, Palo Alto, CA, USA

Full list of author information is available at the end of the article
}

for $12 \%$ of all cases of blindness [1-3]. Patients with DR commonly present with associated vision threatening complications such as diabetic macular edema and neovascularization, which can lead to vitreous hemorrhage and retinal detachment [4]. The probability of developing these complications was shown to be significantly correlated with greater severity of DR [5]. Therefore, monitoring DR severity is crucial for the patient management and 
also an important end-point in several DR clinical trials; the FDA has recently approved the use of ranibizumab in the management of DR [6-8].

The Early Treatment Diabetic Retinopathy Study (ETDRS) stereoscopic 7-field (7F) imaging and grading protocol has been the standard of assessment of DR severity level and used in many DR studies and clinical trials $[6,7,9-14]$. Stereopsis is the perception of depth achieved by merging two slightly different images of the same location utilizing a stereoscopic viewer. In assessing DR severity, the perception of depth is generally presumed to: (1) help to differentiate neovascularization from intraretinal microvascular abnormalities (IRMA);

(2) detect pre-retinal and vitreous hemorrhage; and (3) identify presence of macular edema. Despite these advantages, acquiring and grading stereoscopic 7F photographs are time-consuming, and highly dependent on the experiences of graders and training of photographers $[15,16]$. Additionally, previous study showed that stereoscopic effect may not be critical for the assessment of DR severity [17].

In the recent years, one method developed for viewing the retina in a single shot, while retaining normal resolution of the original monoscopic photographs, is to create a montage by stitching monoscopic photographs together. Many publications in the literature applied montage to describe retinal diseases [18-21]. In a previous study, Li et al. compared assessment of DR severity using a monoscopic auto-mosaic image to standard stereoscopic 7F photographs [22]. In comparing to the montage, the mosaic is created from 9 monoscopic fields, one centered in the macula and others surrounding the macula. Meanwhile, the montage is created from 7 monoscopic fields $[23,24]$. To the best of our knowledge, no one has applied the use of montage image in the assessment of DR severity and compared it to stereoscopic $7 \mathrm{~F}$ images. Therefore, in this study, we want to compare the classification of ETDRS DR severity between stereoscopic 7F and non-stereoscopic montage of monoscopic 7F photographs.

\section{Methods}

The study was conducted in compliance with the Declaration of Helsinki, the US Code of Federal Regulations Title-21, and the Harmonized Tripartite Guidelines for Good Clinical Practice (1996). De-identified images from the Diabetic Retinopathy Repository at the Ocular Imaging Research and Reading Center (OIRRC, Sunnyvale, California) were used for the analysis. Images were from subjects participating in an IRB approved DME clinical trial were utilized for this analysis. Clinical trials used standardized imaging protocol from OIRRC to capture images, and all patients were dilated. Eyes with complications of the posterior pole other than DR, such as age-related macular degeneration (AMD) and posterior uveitis, were excluded from the study. Subjects with media opacities or small pupil size leading to limitations in visualizing the retina were excluded from the analysis to reduce bias in grading.

\section{ETDRS stereoscopic 7-field color fundus photographs}

A total of 16 digital $35^{\circ}$ photographs, seven non-simultaneous color fundus ETDRS stereoscopic 7F pairs and one pair of fundus reflex images, were taken using high-resolution camera. Subjects' pupils were dilated before imaging session. All images were taken by centralized reading center-certified photographers.

\section{Montage images}

Montages were created manually by a trained technician using Adobe Photoshop CS6 Extended (Adobe Systems Incorporated, San Jose, CA). The better image of each stereoscopic pair from stereoscopic 7F photographs was chosen for montage assembly based on illumination, sharpness of blood vessels, and absence of vitreous artifacts. Images were adjusted and aligned manually following blood vessels and other characteristic such as retinal hemorrhages and hard exudates. The "Auto-Blend Layers" tool in the software was utilized to blend images into the montage. An example of ETDRS 7F stereoscopic photographs and the corresponding montage is shown in Fig. 1.

\section{Grading of images}

All images were graded by three certified independent graders $(\mathrm{MH}, \mathrm{NN}$, and $\mathrm{MSH})$ for assessment of $\mathrm{DR}$ severity based on DR severity scale adopted from ETDRS Report 12 [23]. The graders had not participated in any examination of the subjects and were masked to all clinical information about the subjects. All three graders were first asked to perform grading on stereoscopic 7F photographs. Graders then waited at least 14 days before grading the montage images. The purpose of this approach was to prevent recall bias. Stereoscopic 7F photographs and the corresponding montage of each eye were assigned to different code numbers by a fourth team member (SB). The sequence of eyes in the set of stereoscopic 7F photographs was ensured to be different from the set of montages. For grading of stereoscopic $7 \mathrm{~F}$ images, a pair of stereoscopic images for each field was displayed side-by-side on a $4 \mathrm{~K}$ high-resolution monitor and viewed with a Berezin Pocket 3Dvu (Berezin Stereo Photography Products, Mission Viejo, CA) stereoscope viewer. To grade the montage, the image was viewed on the same monitor and zoomed into view each field at the graders' suitable magnification. All graders' assessments 

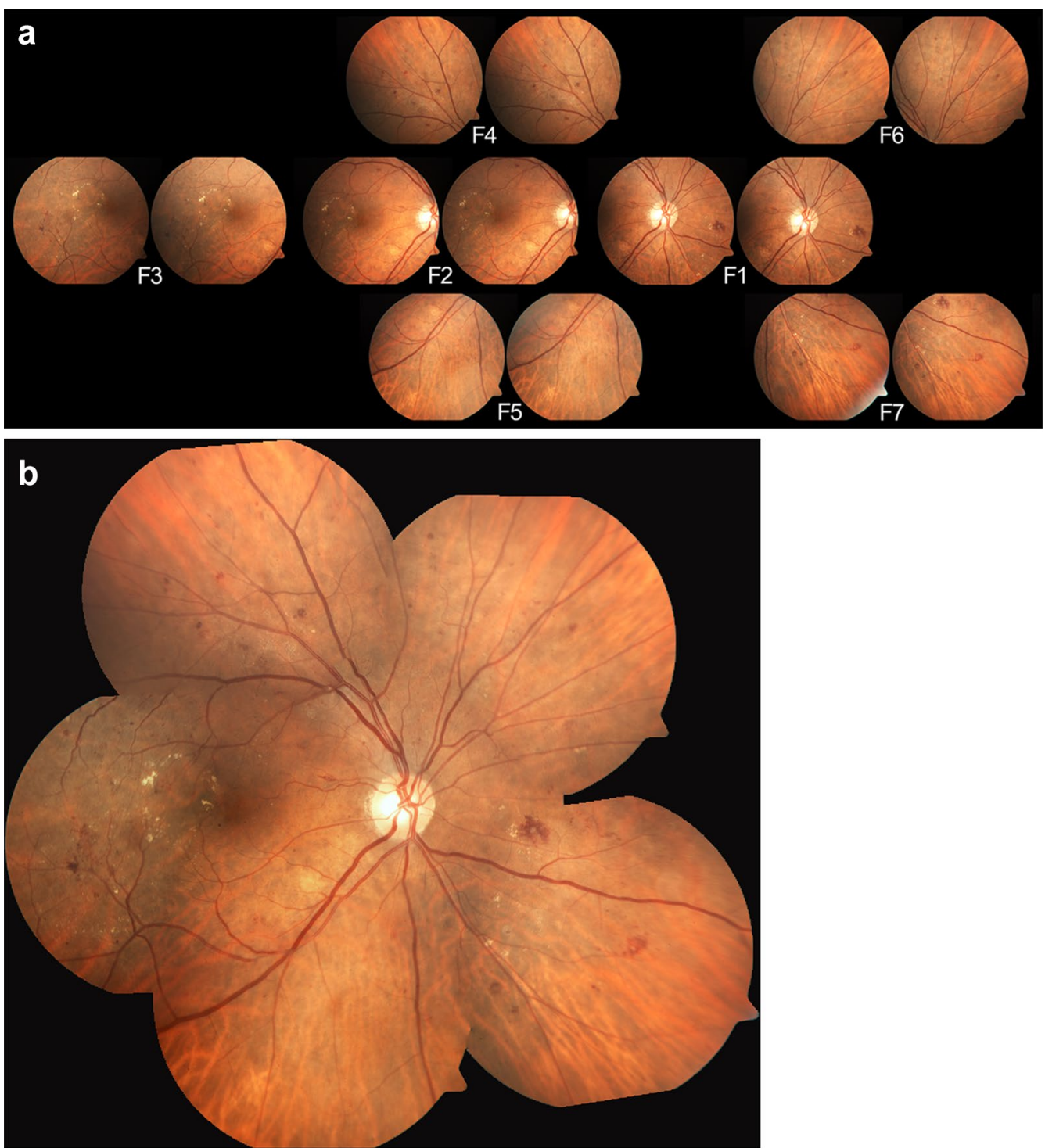

Fig. 1 Example of standard stereoscopic 7-field photographs (a); and the corresponding montage image (b)

of DR severity on stereoscopic 7F photographs and on montages were recorded into a spreadsheet. DR severity level for each eye was adjudicated as the central tendency among three graders. Discrepancies among readers were adjudicated as follows: if two graders agreed, that level was accepted; if all graders differed in grading, the median level was accepted [22].

\section{Statistics}

Diabetic retinopathy severity level agreement between stereoscopic 7F photographs and montage was crosstabulated, and $\mathrm{\kappa}$-value and weighted $\mathrm{\kappa}$-value were calculated to quantify the level of agreement. The $\kappa$-value was interpreted according to guidelines adopted from Landis and Koch [25]:<0.20, poor agreement; 0.21-0.40, fair agreement; 0.41-0.60, moderate agreement; 0.61-0.8, substantial agreement; and 0.81-1.00, perfect agreement. Weighted $\mathrm{k}$-value was utilized to account for the degree of disagreement. The Stuart-Maxwell test of marginal homogeneity was also performed to assess differences in the percentage of severity levels between montage and stereoscopic 7F photographs. Sensitivity, specificity, positive/negative predictive values (PPV/NPV), and positive/negative likelihood ratios (PLR/NLR) for montage grading method were calculated using the grading of DR severity on stereoscopic 7F photographs as reference. 
$\mathrm{p}<0.05$ was considered significant on all tests in the analysis. Statistical analyses were performed using Stata, version 14.2 (Stata Corp LLC, College Station, Texas, USA).

\section{Results}

Baseline characteristics

A total of 50 eyes, 32 right and 18 left, were included in the study. The median DR severity score was moderately severe NPDR (level 47) on both stereoscopic 7F and montage images. The distribution of DR severity level assessed by stereoscopic 7F photographs was level 10 (0 eye); level 14/15/20 (0 eye); level 35 ( 6 eyes); level 43 (11 eyes); level 47 (13 eyes); level 53 (7 eyes); and level $\geq 60$ (13 eyes).

Stereoscopic 7F and montage agreement of severity levels DR severity agreement between $7 \mathrm{~F}$ and montage was cross-tabulated in Table 1 . There was a substantial agreement between stereoscopic $7 \mathrm{~F}$ and montage $(\kappa=0.745$, $\left.\kappa_{\text {weighted }}=0.867, \mathrm{p}<0.0001\right)$ in the assessment of DR severity score. Of 50 eyes, 40 (80\%) eyes showed complete agreement, and $100 \%$ of the cases had agreement within 1-step (Table 2). The difference in percentage of DR severity levels between stereoscopic $7 F$ and montage was not statistically significant $(\mathrm{p}=0.6151)$.

\section{Comparison of stereoscopic 7F and montages at different severity levels}

The agreement in DR severity assessment at different DR severity levels between stereoscopic 7F photography and montage was shown in Table 3. The rate of agreement between stereoscopic $7 \mathrm{~F}$ and montages ranged from 0.88 to 1.00 at different severity levels with the lowest at level 35 (mild NPDR).
Table 2 Level of agreement for the assessment of ETDRS DR severity score on stereoscopic 7F compared with montage

\begin{tabular}{ll}
\hline & $\begin{array}{l}\text { Montage } \\
\text { vs. 7-field } \\
\text { stereoscopic }\end{array}$ \\
\hline Complete agreement & $80(\%)$ \\
Agreement within 1-step & $100(\%)$ \\
K-value & $0.745(p<0.0001)$ \\
Weighted k-value & $0.867(p<0.0001)$ \\
\hline
\end{tabular}

Sensitivity, specificity, positive/negative predictive values of the montage grading method

Sensitivity, specificity, positive/negative predictive values, and positive/negative likelihood ratio for montage at different severity levels were shown in Table 3. In comparing montage with stereoscopic $7 \mathrm{~F}$ photographs, the sensitivity ranged from 0.33 to 1.00 at different severity levels. The lowest sensitivity was at level 35 (mild NPDR). Specificity and NPV for montage were similar across all severity levels. PPV for montage ranged from 0.50 to 1.0 , and the lowest PPV was at level 35. PLR for montage at level 47 (moderately severe NPDR) (25.67) was higher than other levels that can be explained by high specificity at this level. Because there was a complete agreement at level $\geq 60$ (PDR) between stereoscopic 7F and montages, PLR at this level was not able to be calculated. NLR for montage at level 53 (severe NPDR) and level $\geq 60$ was zero because sensitivity at these levels was equal to 1 .

\section{Intergrader agreement}

Intergrader agreement was similar on both stereoscopic $7 \mathrm{~F}$ and montages. The intergrader $\mathrm{\kappa}$ and weighted

Table 1 ETDRS DR severity score assessed from grading 7-field stereoscopic photographs compared to montage images

\begin{tabular}{|c|c|c|c|c|c|c|c|c|}
\hline & \multicolumn{8}{|c|}{ Montage } \\
\hline & 10 & $14,15,20$ & 35 & 43 & 47 & 53 & $\geq 60$ & Total \\
\hline \multicolumn{9}{|c|}{ Stereoscopic 7F photographs } \\
\hline 10 & & & & & & & & 0 \\
\hline 14,15 and 20 & & & & & & & & 0 \\
\hline 35 & & & 2 & 4 & & & & 6 \\
\hline 43 & & & 2 & 8 & 1 & & & 11 \\
\hline 47 & & & & 1 & 10 & 2 & & 13 \\
\hline 53 & & & & & & 7 & & 7 \\
\hline$\geq 60$ & & & & & & & 13 & 13 \\
\hline Total & 0 & 0 & 4 & 13 & 11 & 9 & 13 & 50 \\
\hline
\end{tabular}

Level 10: DR Absent; 14 and 15: DR Questionable; 20: Microaneurysms Only; 35: Mild NPDR; 43: Moderate NPDR; 47: Moderately Severe NPDR; 53: Severe NPDR; 260 : PDR 
Table 3 Diabetic retinopathy severity level: stereoscopic 7F photographs compared with montage image

\begin{tabular}{llllllll}
\hline Retinopathy severity & Sensitivity & Specificity & PPV & NPV & PLR & $\begin{array}{l}\text { NLR } \\
\text { Rate } \\
\text { of agreement }\end{array}$ \\
\hline Level 35 & 0.33 & 0.95 & 0.50 & 0.91 & 6.60 & 0.71 & 0.88 \\
Level 43 & 0.73 & 0.87 & 0.62 & 0.92 & 5.62 & 0.31 & 0.91 \\
Level 47 & 0.77 & 0.97 & 0.91 & 0.92 & 25.67 & 0.24 & 0.91 \\
Level 53 & 1.00 & 0.95 & 0.78 & 1.00 & 20.00 & 0.00 & 0.95 \\
Level $\geq 60$ & 1.00 & 1.00 & 1.00 & 1.00 & - & 0.00 & 1.00 \\
\hline
\end{tabular}

PPV positive predictive value, NPV negative predictive value, $P L R$ positive likelihood ratio, NLR negative likelihood ratio

K-values were shown in Table 4 . There was a moderate agreement ( $\mathrm{k}$-value ranging from 0.4705 to 0.5803 , $\mathrm{p}<0.0001)$ between graders on both montage and stereoscopic $7 \mathrm{~F}$ photographs. The weighted $\mathrm{k}$-value ranged from 0.6511 to $0.7472, \mathrm{p}<0.0001$.

\section{Discussion}

Assessing severity of DR is important for both patient management and outcome measure in DR clinical trials. The early treatment diabetic retinopathy severity stereoscopic 7F photography imaging and grading protocol has been a gold standard for assessment of DR severity level and used in many DR clinical trials [6, 7, 9-14, 23, 24]. In this study, we compared assessment of DR severity between stereoscopic 7F photographs and montage image.

The results of our study suggest that montage image is comparable to ETDRS stereoscopic 7F photographs for assessment of DR severity. Previously, $L i$ et al. employed a similar three-grader system to compare monoscopic mosaic image to standard stereoscopic 7F photographs for grading DR severity [22]. In their study, there was a substantial agreement between the mosaic and stereoscopic 7F photographs $\left(\kappa=0.62, \kappa_{\text {weighted }}=0.86\right)$ for grading DR severity. Similar findings were also found between montage and stereoscopic 7F photographs in our study $\left(\kappa=0.745, \kappa_{\text {weighted }}=0.867\right)$. Similarly, they noted complete agreement between the graders in $66.9 \%$ of images and agreement within one-step in $97.4 \%$ of the cases. In

Table 4 Intergrader diabetic retinopathy severity level agreement on stereoscopic 7 fields and montages

\begin{tabular}{|c|c|c|c|}
\hline & Stereoscopic 7F $(n=50)$ & Montage $(n=50)$ & ETDRS report 12 \\
\hline Complete agreement (\%) & & & $53^{\mathrm{a}}$ \\
\hline Grader MH vs NN & 64 & 58 & \\
\hline Grader MH vs MSH & 64 & 58 & \\
\hline Grader NN vs MSH & 64 & 68 & \\
\hline Agreement within one step (\%) & & & $88^{\mathrm{a}}$ \\
\hline Grader MH vs NN & 98 & 98 & \\
\hline Grader MH vs MSH & 94 & 96 & \\
\hline Grader NN vs MSH & 92 & 90 & \\
\hline \multicolumn{4}{|l|}{ Agreement within two steps (\%) } \\
\hline Grader MH vs NN & 100 & 100 & \\
\hline Grader MH vs MSH & 96 & 96 & \\
\hline Grader NN vs MSH & 96 & 96 & \\
\hline k-value & & & $0.42^{a}$ \\
\hline Grader MH vs NN & $0.4705(p<0.0001)$ & $0.5403(p<0.0001)$ & \\
\hline Grader MH vs MSH & $0.4710(p<0.0001)$ & $0.5434(p<0.0001)$ & \\
\hline Grader NN vs MSH & $0.5803(p<0.0001)$ & $0.5452(p<0.0001)$ & \\
\hline Weighted k-value & & & $0.65^{\mathrm{a}}$ \\
\hline Grader MH vs NN & $0.7153(p<0001)$ & $0.7472(p<0.0001)$ & \\
\hline Grader MH vs MSH & $0.6511(p<0.0001)$ & $0.6975(p<0.0001)$ & \\
\hline Grader NN vs MSH & $0.7153(p<0001)$ & $0.6873(p<0.0001)$ & \\
\hline
\end{tabular}

\footnotetext{
a These intergrader agreement values were obtained from ETDRS Report 12
} 
contrast, we noted a higher level of complete agreement (80\%) and agreement within one-step (100\%). The differences may be due to several reasons. Even though the mosaic image covered a larger area than the corresponding 7F photographs, it did not include entirely 7F retinal area. Moreover, the auto-mosaic feature of the algorithm did not choose the better-quality view when assembling the composite image. On the other hand, the montage images used in our study was assembled manually by a trained technician using the better-quality image of each stereoscopic pair based on certain criteria (illumination, sharpness of blood vessels, and absence of vitreous artifacts). We also utilized the "Auto-Blend Layers" tool in Photoshop masked out underexposed area in the overlapping regions and yielded a smooth transition in the final composite montage image.

Several studies have compared ultra-widefield (UWF) image and monoscopic 7F photographs to stereoscopic 7F photographs in the assessment of DR severity level in the literature $[15,17,26,27]$. Although the UWF images provide larger view of the retina, the stereoscopic $7 \mathrm{~F}$ photographs have higher resolution than UWF images. Therefore, the 7F photographs, which have the same resolution as montage, provide advantages for identifying small lesions. Aiello et al. have demonstrated that UWF images have lower sensitivity in identifying certain retinopathy lesions compared to $7 \mathrm{~F}$ photographs [27]. Moreover, the UWF images provide no real color images, but only two monochromatic red and green SLO scans, resulting in semirealistic fundus images [15]. UWF imaging equipment is also not readily available, and until the day UWF cameras become the norm, we will need to rely on conventional fundus photography to evaluate DR. Advantages and disadvantages of different imaging methods in assessing DR severity are summarized in Table 5.

We analyzed the sensitivity, specificity, PPV, NPV, PLR, and NLR for montage grading methodology using stereoscopic 7F grading as a standard (Table 3 ). The montage grading methodology was found to be highly specific at all DR severity levels with a very high negative predictive value. However, there was a variation in terms of sensitivity of this grading methodology. The sensitivity of the methodology was lower at level 35 (31\%) but significantly increased to $>70 \%$ at level 43 and 47 and reached $100 \%$ at $\geq$ Level 53 and above. The stereoscopic $7 \mathrm{~F}$ photographs have a certain degree of overlap between the adjacent fields. Therefore, some lesions are usually seen in multiple fields. The advantage of such approach is that graders can use different views of same lesion to confirm their findings. However, the disadvantage is that the same lesion on multiple fields can potentially be counted as two different occurrences and give rise to a different severity score. The monoscopic montage image, on the other hand, decreases the chances of counting a single lesion twice since the entire 7 field area is visible together. Even though the "Auto-Blend Tool" allows a smooth evenly exposed image, it sometimes may result in over or under enhancement of an area. These differences in the montage grading and stereoscopic $7 \mathrm{~F}$ grading methodologies can potentially explain the variation in sensitivities that we noted in our study.

The intergrader agreement for assessment of DR severity based on both montage and stereoscopic $7 \mathrm{~F}$ imaging methodology in or study was comparable to other studies including the ETDRS Report 12 (Table 4) [17, 22, 23]. In the ETDRS Report 12, complete agreement between graders occurred $53 \%$ of the time, and

Table 5 Advantages and disadvantages of different imaging methods for screening and assessment of DR severity

\begin{tabular}{|c|c|c|}
\hline Imaging methods & Advantages & Disadvantages \\
\hline Stereoscopic 7-field & $\begin{array}{l}\text { Stereopsis for detecting DME, NVE, NVD, pre-retinal } \\
\text { hemorrhages, and vitreous hemorrhages }\end{array}$ & $\begin{array}{l}\text { Time-consuming process and requires highly training photographers for } \\
\text { capturing images }[15,16] \\
\text { Requires stereo viewer while grading images in order to appreciate stereo } \\
\text { effect } \\
14 \text { images are captured }\end{array}$ \\
\hline Montage & $\begin{array}{l}\text { Viewing } 7 F \text { area in a single shot while maintaining } \\
\text { original monoscopic images } \\
\text { Less photographs are taken (7 total) }\end{array}$ & $\begin{array}{l}\text { Time-consuming in constructing montage and requires highly training } \\
\text { technician } \\
\text { Lack of stereopsis }\end{array}$ \\
\hline Monoscopic 7-field & Less photographs are taken (7 total) & Lack of stereopsis \\
\hline Mosaic & Less photographs are taken (9 total) & $\begin{array}{l}\text { Lack of stereopsis } \\
\text { Uneven transition between adjacent fields [22] } \\
\text { Does not entirely cover 7F area although covers larger retinal area [22] }\end{array}$ \\
\hline Ultra-widefield & $\begin{array}{l}\text { Only one photograph is taken } \\
\text { Covers much larger retinal area } \\
\text { Viewing retinal area in a single shot } \\
\text { Great screening tool for the presence of DR }[15,26]\end{array}$ & $\begin{array}{l}\text { Lack of stereopsis } \\
\text { Lower sensitivity in detecting certain retinopathy lesions }[26,27] \\
\text { Semirealistic fundus images [15] }\end{array}$ \\
\hline
\end{tabular}




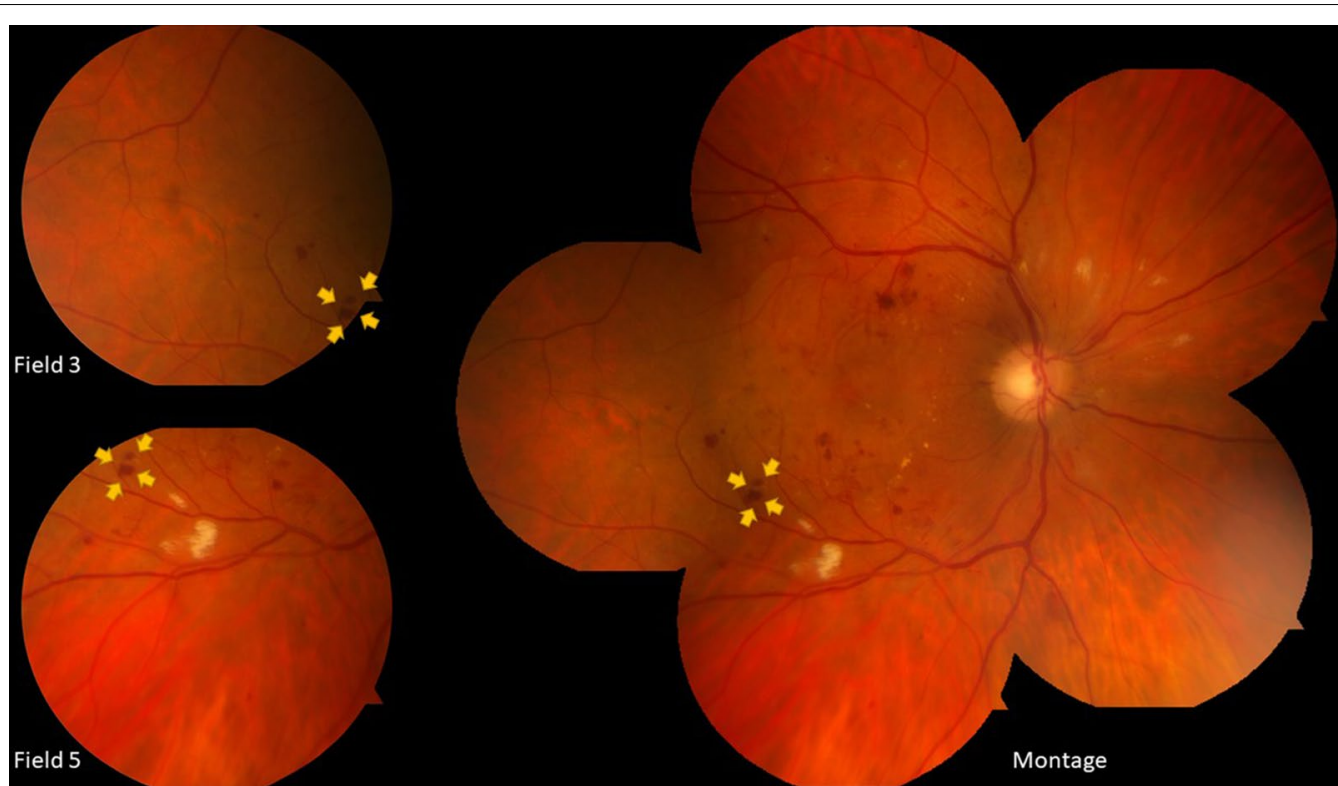

Fig. 2 The retinal hemorrhage (yellow arrows) in Field 3 and Field 5 can be mistakenly counted as two occurrences. Meanwhile, this retinal hemorrhage is counted as only one occurrence on the montage

the $\mathrm{k}$-value was 0.42 [23]. In this study, complete agreement occurred on $64 \pm 0 \%$ of stereoscopic $7 \mathrm{~F}$ images, and $61 \pm 5.8 \%$ of montage, and the average $\kappa$-value was 0.51 and 0.54 on stereoscopic $7 \mathrm{~F}$ and montage, respectively.

Considering recent developments of the artificial intelligence algorithms for detection and diagnosis of DR characteristics, montage images may have the advantage of allowing better and more efficient lesion quantification by these algorithms, especially in terms of decreasing the chances of counting same lesion as two occurrences (Fig. 2).

While montage grading methodology appears to be comparable to stereoscopic 7F photographs in assessing DR severity level, our study does have its limitations. The study had a small sample size, and variability of the subjects did not cover the entire spectrum of EDTRS DR severity scale. There was low frequency of PDR lesions (NVD, fibrous proliferations on the disc, and $\mathrm{VH}$ ), and a small number of subjects with DR severity equal or less than level 35. In addition, construction of the montage is still a time-consuming process, and the technician was required to complete an intensive training process to be certified for montage construction. Another disadvantage of montage image is that due to its lack of stereopsis, the montage is less likely to provide the ability to detect and grade diabetic macular edema (DME) as compared to stereoscopic 7F photographs. However, presence or absence of DME does not impact the DR severity level and its assessment was not included in this study.

\section{Conclusions}

In conclusion, we have found a substantial agreement in assessing ETDRS DR score on montage and stereoscopic 7F photographs. Intergrader agreement was also comparable in this study compared to other studies. Therefore, montage of the 7 fields can be used confidently as a possible and time-saving alternative imaging method to stereoscopic 7F photographs in assessing DR severity level in clinical research.

\section{Abbreviations}

DR: Diabetic retinopathy; ETDRS: Early Treatment Diabetic Retinopathy Study; IRMA: intraretinal microvascular abnormalities; AMD: age-related macular degeneration; PLR: positive likelihood ratio; NLR: negative likelihood ratio; PPV: positive predictive value; NPV: negative predictive value; NPDR: non-proliferative diabetic retinopathy; PDR: proliferative diabetic retinopathy; NVD: new vessels in disc.

\section{Acknowledgements}

Not applicable.

\section{Authors' contributions}

NN: Study concept and design, acquisition of data, analysis and interpretation, manuscript drafting and revising. EV: Study concept and design, data analysis and interpretation, manuscript drafting and revising. MH: Study design, data interpretation, manuscript review and revision. MSH: Data interpretation, data analysis. SB: Acquisition of data. HG: Acquisition of data. RA: Acquisition of data, manuscript writing. DVD: Study concept, and interpretation of data. YJS: Study concept and design, manuscript revision, final approval to publish. All authors read and approved the final manuscript. 


\section{Funding}

DVD has received research funding support from Genentech and Regeneron and has served on the scientific advisory boards for Allergan, Clearside, Genentech, and Regeneron. YJS has received research support from Astellas, Genentech and Optovue, and has served as a consultant for Optos.

\section{Availability of data and materials}

The datasets used during the current study are available from the corresponding author on request.

\section{Ethics approval and consent to participate}

The study was conducted in compliance with the Declaration of Helsinki, the US Code of Federal Regulations Title-21, and the Harmonized Tripartite Guidelines for Good Clinical Practice (1996).

\section{Consent for publication}

Not applicable.

\section{Competing interests}

The authors declare that they have no competing interests.

\section{Author details}

${ }^{1}$ Byers Eye Institute, Stanford University, 2370 Watson Court, Suite 200, Palo Alto, CA, USA. ${ }^{2}$ Ocular Imaging Research and Reading Center, Sunnyvale, CA USA. ${ }^{3}$ University of Texas Southwestern School of Medicine, Dallas, TX, USA. ${ }^{4}$ College of Arts and Sciences, University of Nebraska-Lincoln, Lincoln, NE, USA.

Received: 3 April 2019 Accepted: 25 November 2019 Published online: 13 December 2019

\section{References}

1. Engelgau MM, Geiss LS, Saaddine JB, et al. The evolving diabetes burden in the United States. Ann Intern Med. 2004;140(11):945-50.

2. Lee R, Wong TY, Sabanayagam C. Epidemiology of diabetic retinopathy, diabetic macular edema and related vision loss. Eye Vis (Lond). 2015;2:17.

3. Wong TY, Klein R, Islam FM, et al. Diabetic retinopathy in a multi-ethnic cohort in the United States. Am J Ophthalmol. 2006;141(3):446-55.

4. El Annan J, Carvounis PE. Current management of vitreous hemorrhage due to proliferative diabetic retinopathy. Int Ophthalmol Clin. 2014;54(2):141-53.

5. Klein R, Klein BE, Moss SE, Cruickshanks KJ. The Wisconsin Epidemiologic Study of Diabetic Retinopathy: XVII. The 14-year incidence and progression of diabetic retinopathy and associated risk factors in type 1 diabetes. Ophthalmology. 1998;105(10):1801-15.

6. Hassan M, Sadiq MA, Halim MS, Afridi R, Nguyen NV, Sepah YJ. Short-term effects of ranibizumab on diabetic retinopathy severity and progression. Ophthalmol Retina. 2018;2(7):749-51.

7. Ip MS, Domalpally A, Hopkins JJ, Wong P, Ehrlich JS. Long-term effects of ranibizumab on diabetic retinopathy severity and progression. Arch Ophthalmol. 2012;130(9):1145-52.

8. Writing Committee for the Diabetic Retinopathy Clinical Research N, Gross JG, Glassman AR, et al. Panretinal photocoagulation vs intravitreous ranibizumab for proliferative diabetic retinopathy: a randomized clinical trial. JAMA. 2015:314(20):2137-46.

9. Epidemiology of Diabetes Interventions and Complications (EDIC). Design, implementation, and preliminary results of a long-term follow-up of the Diabetes Control and Complications Trial cohort. Diabetes Care. 1999;22(1):99-111.

10. The Diabetes Control and Complications Trial (DCCT). The Diabetes Control and Complications Trial (DCCT) Design and methodologic considerations for the feasibility phase. The DCCT Research Group. Diabetes. 1986;35(5):530-45.

11. Chew EY, Ambrosius WT, Howard LT, et al. Rationale, design, and methods of the Action to Control Cardiovascular Risk in Diabetes Eye Study (ACCORD-EYE). Am J Cardiol. 2007:99(12A):103i-11i.
12. Pieramici DJ, Wang PW, Ding B, Gune S. Visual and anatomic outcomes in patients with diabetic macular edema with limited initial anatomic response to ranibizumab in RIDE and RISE. Ophthalmology. 2016;123(6):1345-50.

13. Wykoff CC, Eichenbaum DA, Roth DB, Hill L, Fung AE, Haskova Z. Ranibizumab induces regression of diabetic retinopathy in most patients at high risk of progression to proliferative diabetic retinopathy. Ophthalmol Retina. 2018;2(10):997-1009.

14. Lim RR, Vaidya T, Gadde SG, et al. Correlation between systemic S100A8 and S100A9 levels and severity of diabetic retinopathy in patients with type 2 diabetes mellitus. Diabetes Metab Syndr. 2019;13(2):1581-9.

15. Kernt M, Hadi I, Pinter F, et al. Assessment of diabetic retinopathy using nonmydriatic ultra-widefield scanning laser ophthalmoscopy (Optomap) compared with ETDRS 7-field stereo photography. Diabetes Care. 2012;35(12):2459-63.

16. Rasmussen ML, Broe R, Frydkjaer-Olsen U, et al. Comparison between Early Treatment Diabetic Retinopathy Study 7-field retinal photos and non-mydriatic, mydriatic and mydriatic steered widefield scanning laser ophthalmoscopy for assessment of diabetic retinopathy. J Diabetes Complications. 2015:29(1):99-104

17. Li HK, Hubbard LD, Danis RP, Esquivel A, Florez-Arango JF, Krupinski EA. Monoscopic versus stereoscopic retinal photography for grading diabetic retinopathy severity. Invest Ophthalmol Vis Sci. 2010;51 (6):3184-92.

18. Casalino G, Bandello F, Chakravarthy U. An Unusual Cause of Unilateral Vision Loss. JAMA Ophthalmol. 2017;135(1):69-70.

19. Kumar N, Sudharshan S, Ganesh SK, Lingam G, Biswas J. Bilateral multiple choroidal granulomas and systemic vasculitis as presenting features of tuberculosis in an immunocompetent patient. J Ophthalmic Inflamm Infect. 2016:6(1):40.

20. Saurabh K, Das RR, Biswas J, Kumar A. Profile of retinal vasculitis in a tertiary eye care center in Eastern India. Indian J Ophthalmol. 2011;59(4):297-301.

21. Witkin AJ, Shah AR, Engstrom RE, et al. Postoperative Hemorrhagic Occlusive Retinal Vasculitis: expanding the Clinical Spectrum and Possible Association with Vancomycin. Ophthalmology. 2015;122(7):1438-51.

22. Li HK, Esquivel A, Hubbard LD, Florez-Arango JF, Danis RP, Krupinski EA. Mosaics versus Early Treatment Diabetic Retinopathy seven standard fields for evaluation of diabetic retinopathy severity. Retina. 2011;31(8):1553-63.

23. Fundus photographic risk factors for progression of diabetic retinopathy. Fundus photographic risk factors for progression of diabetic retinopathy ETDRS report number 12. Early Treatment Diabetic Retinopathy Study Research Group. Ophthalmology. 1991;98(5 Suppl):823-33.

24. Early Treatment Diabetic Retinopathy Study Research Group. Grading diabetic retinopathy from stereoscopic color fundus photographs-an extension of the modified Airlie House classification. ETDRS report number 10 Early Treatment Diabetic Retinopathy Study Research Group. Ophthalmology. 1991;98(5 Suppl):786-806.

25. Landis JR, Koch GG. The measurement of observer agreement for categorical data. Biometrics. 1977;33(1):159-74

26. Aiello LP, Odia I, Glassman AR, et al. Comparison of Early Treatment Diabetic Retinopathy Study standard 7-field imaging with ultrawide-field imaging for determining severity of diabetic retinopathy. JAMA Ophthalmol. 2019;137(1):65-73.

27. Silva PS, Cavallerano JD, Sun JK, Noble J, Aiello LM, Aiello LP. Nonmydriatic ultrawide field retinal imaging compared with dilated standard 7-field 35-mm photography and retinal specialist examination for evaluation of diabetic retinopathy. Am J Ophthalmol. 2012;154(3):549-59.

\section{Publisher's Note}

Springer Nature remains neutral with regard to jurisdictional claims in published maps and institutional affiliations. 